cases ten minims of tincture of belladonna were substituted for the tincture of hyoscyamus, but without special benefit. After the bowels had been thoroughly opened, iodide of potassium in five-grain doses, in infusion of quassia, was given three times a day. If any epigastric pain continued, preventing sleep, opium in grain or half-grain doses was ordered at bedtime, as necessary. When any tendency to constipation remained, colocynth-pill and hyoscyamus was given from time to time. Under the influence of the iodide the tongue cleaned, appetite returned, and, in fact, all the symptoms gradually subsided; though a sense of sinking with occasional epigastric pain sometimes persisted for a long time, and strength was only very slowly regained.

Some of the milder cases were treated from the first with half-drachm doses of aromatic sulphuric acid. The improvement under its use was not so steady as under the iodide.

One case was treated in the first instance with scruple doses of alum every three hours. This treatment was abandoned after two drachms had been taken, as the pain had increased in severity and become unbearable, the bowels remaining confined. There had been no motion from the bowels for four days before the alum treatment was begun, and an evacuation was only obtained on the fifth day, after two ounces and a half of Epsom salts had been taken. Morphia had in the meantime been ordered, to relieve the epigastric pain. Great and permanent relief followed the action of the purgative. He was then placed on theiodide, and did well.

The experience gained in this case did not bear out the strong statements which have been made in favour of alum as a remedy for lead-poisoning.

Bromide of potassium was given in one case in ten-grain doses, three times a day, with apparent benefit, the patient having in the first instance been freely purged by sulphate of magnesia. In this case the improvement was as great and as rapid as in the cases under the iodide. It cannot, however, be said that it was more so.

The following experiment was instituted for the purpose of ascertaining the comparative solvent powers possessed by iodide and bromide of potassium for the salts of lead. Eighty grains each of iodide and bromide of potassium were respectively dissolved in two drachms of distilled water, at $84^{\circ}$ Fahr. It had been intended to perform the experiment at blood-heat, but at the conclusion of the process the temperature stood as stated. A solution of acetate of lead in distilled water, containing one grain of the salt in every three minims, was added till the precipitate ceased to be redissolved. The point of saturation was reached when fourteen minims of the lead solution had been added to the iodide; while in the case of the bromide fifty-four minims were required before the same point was attained. In other words, eighty grains of iodide of potassium in two drachms of water, at $84^{\circ} \mathrm{Fahr}$, held in solution only $4 \frac{2}{3}$ grains of the acetate of lead; while an equivalent preparation of the bromide of potassium beld in solution eighteen grains of the same salt.

On theoretical grounds, therefore, we might conclude that the bromide of potassium would be a better remedy in cases of chronic lead-poisoning than the iodide of potassium, more especially if we take into account the influence over the nervous system possessed by the bromide, as shown by its power of procuring sleep in some cases of disease. This remedy would at least be worthy of a more extended trial, in doses of not less than fifteen grains, three times a day.

The chemical treatment of disease has not always been a happy one, and nothing but an extended and carefully recorded clinical experience will justify emphatic statements in favour of any particular remedy.

It may be observed that in some cases the blue line on the margin of the gums was most persistent, remaining after all the other symptoms had long subsided. When such was the case the treatment was continued for some weeks after the man bad returned to duty. The depth of this blue line, or its intensity, bears no fixed proportion to the severity of the epigastric pain; yet it may be taken as a fair index of the degree of poisoning, as shown by the other symptoms; those who presented the deepest blue line being the most debilitated and remaining longest under treatment.

So soon as the first case amongst the shipwrights came under notice, Dr. Saunders, staff-surgeon, recommended that the men should not work at scaling, or be in any way connected with paint, for more than three days in any week, and that on the other three days they should have open-air employment. A number of simple precautions were also issued for the guidance of the men. The necessity of perfect cleanliness was minutely dwelt upon, the working dress directed to be frequently washed, and always removed before eating; a veil recommended to be worn over the nose and mouth whilst at work; and fat and oily substances to be added to the diet as much as possible. An agreeable and inexpensive drink, composed of water acidulated with sul. phuric acid and flavoured with orange-peel, was suggested to be always kept in readiness to quench thirst. Under these improved conditions the number of cases shortly subsided, and such as did occur were less severe in character. The men themselves willingly adopted the suggestions, inventing various respirators for themselves, such as picked oakum, pieces of sponge sewn between muslin, as well as veils of different textures, \&c. There is a feeling amongst the men in favour of the oakum respirators, and an im. pression exists amongst the workmen that those with thick beards and moustaches suffer least from the effects of the lead.

In contrasting these cases with those of the first series, it will be seen to how much greater a degree the poisoning had extended in each case, and that, though perhaps the effects of the lead were intensified by working in a close atmosphere, the symptoms were not wholly to be accounted for by the prolonged and repeated breathing of impure air, as they might be in some of the cases of the first series, considering how quickly the symptoms passed off. In the first series the treatment usually lasted under one week, in the second series from three to eight weeks.

\section{ON THE TREATMENT OF IMPASSABLE STRICTURE.*}

By W. F. TEEVAN, B.A., F.R.C.S.,

SURGEON TO THE WEST LONDON HOSPITAL, SURGEON TO ST. PETTR'S HOSPITAL, AND FORMBRLY LECTURER ON ANATOMY AT THEA WESTMINSTER HOSPITAL.

I HAVE used the word "impassable" as not only being more correct, but as conveying to our minds a certain definite fact more clearly than the word "impermeable." A patient's contracted urethra may permit the escape of urine but forbid the entry of any instrument. It is now, $I$ think, universally admitted that, excepting cases from injury, no urethra is ever obliterated, and therefore urine never ceases to flow in varying quantities unless its course be diverted by flowing into a fistulous channel. Now, this is a most important pathological fact, very prominently brought before the profession ky the late $\mathrm{Mr}$. Syme, and although he modified his celebraled dictum to the effect that "if urine comes out an instrument can get in," yet there remains the great fact that his enunciation of a truth did much to encourage surgeons to persevere in attempting to pass an instrument.

I will now suppose a case of stricture in which the surgeon in attendance is unable to get in any instrument, and I will take it for granted that we are called in to treat the case under those conditions that usually obtain in this country. What is the usual statement made? The practitioner informs us that he has tried to pass the smallest silver catheter without success. I am not at all surprised, for he has by no means exhausted the surgical armoury, and, fortunately, there are instruments in reserve in com. parison to which bis smallest catheter is large. It is clear, therefore, that up to this stage want of success might have been entirely owing to the large size of the instrument employed. The first thing I do is to examine the patient's perineum. If it be in a normal condition I take one of the smallest filiform bougies, but if there be much induration I select a whalebone one. Having placed the patient with his back against the wall, I take my seat in front of him and try to pass the bongie through the stricture. What is * Read before the meeting of the British Medical Association, August 8 th, 1872 . 
the best method to adopt to get it in? I know of no better word than "wriggle" to express the required action. We must wriggle in the bougie. These small bougies sometimes give trouble from their catching in the lacunæ, but we can remedy this inconvenience by withdrawing them for a short distance, and then passing them on again, rotating them as we proceed. Arrived at the face of the stricture, we had first better touch it all over with the instrument to see if we can find the entrance, and if that does not succeed we must then try to screw it in. If a quarter of an hour's trial fail, I then withdraw the bougie and give it that peculiar twist at the end which makes it resemble a skeleton key. I may say that this practice is of old repute in Paris. Should this not prove successfnl after a ten minutes' trial, I introduce one of Leroy D'Etiolles" "bougies tortillées." I usually select one that resembles a corkscrew. If these means fail I do nothing more for two days. I would here remark that my observations on the treatment of impassable stricture refar to that complaint when uncomplicated with retention. I desire the patient to get his bowels thoroughly well open the morning he comes to me, and before I commence the trial I request the patient to make water; this is very important, for the stream of urine washes away any plug of mucus that may be obstructing the passage, and leaves the bladder in the most favourable condition for avoiding any creation, by instrumentation, of a desire to micturate. If on this occasion I fail, after repeating for one half-hour the methods I have already described, I do nothing more for two days.* At the next visit I modify my plan of action. It often happens that the reason why we cannot get in an instrument is, that we are unable to find the entrance to the stricture-it is so small. Now, if we pass a full-sized bougie down to the stricture and keep it there for a quarter of an hour, pressing it all the time against the stricture, we shall distend the parts, and the result will be that the mouth of the stricture will become opened, and we shall probably succeed in getting in a small filiform bougie. I have rarely found this plan fail. I am indebted to M. Mercier for it, and I would refer to his "Rech. de 1845, p. 86, Gaz. Méd." The introduction, by me I believe, of these small French instruments into practice in this country has rendered passable most of the cases which were formerly impassable.

I would here recall to the minds of those present $M$. Mercier's exposition of the pathology of stricture. Stricture is atrophy, not hypertrophy, and not only is the urethra contracted, but it is tortuous. Now, it is most important for us to remember this latter fact, for it shows that as a rule metallic instruments are not suitable for the treatment of tight or impassable strictures, and I think it would be well for the patient if every surgeon remembered M. Mercier's celebrated dictum, "La sonde rigide ne cede pas, c'est l'obstacle qui cede devant elle." No doubt some gentleman has already said to himself, "How is it you do not put the patient under chloroform when you find you cannot get in an instrument?" I will tell you why. Some time ago M. Borovitch, of Tamboff, was over here, and we discussed the subject of impassable stricture. He said to me, "Do you ever give chloroform for retention, or impassable stricture?" I said "No." He then said, "Why not ?" I said, "For the best of all reasons; I never found it of the slightest use." "Exactly so," he said, "it is absolutely useless to any surgeon who is experienced in passing instruments." If a surgeon cannot pass the instrument without chloroform, he cannot do it with, for the reason that chloroform does not lessen the bulk of the tissues composing the stricture and its surroundings. M. Borovitch informed me that if he found a stricture impassable, he put half a dozen leeches on the perineum, and allowed them to bleed freely. He rarely found this plan fail; but if it did, he put the patient into a hot bath, and bled bim till he was faint. This treatment succeeds by enlarging the constricted urethra, for you lessen the quantity of blood in the canal, and you also take off the pressure from the outside. From personal experience of leeching, I can say that I have seldom seen it fail.

I will now suppose that we have exhausted every means to pass an instrument, and have failed. The patient becomes more and more troubled, and an operation is indicated

* The injecting the urethra with oil I have seldom found useful. For an account of this method, introduced by Soemmering, see p. 157 of Perrève' to save or prolong life. What is the best kind of operation to select? There are three operations open to us. First, we have the puncture of the bladder through the rectum, which is the favourite procedure in this coustry. Next, we bave Syme's operation for impermeable urethra. And, lastly, we have the old French operation called "la boutonnière," which, I believe, is but little known in this country, except Leeds. I will now state my objections to the first two. Those surgeons who resort to tapping the bladder through the rectum do so on the ground that if they divert all the urine from the natural channel, and allow it to flow in another, they thereby give the urethra a rest, the irritation and inflammation will subside, and in about a month or six weeks they will be enalled, by the rest they have afforded the urethra, to pass an instrument, and cure the patient by gradual dilatation. Now, there are no less than four grave objections to this operation. In the first place, the operation can only be employed in those cases where there is no enlargement of the prostate; secondly, the surgeon may fail to pass an instrument through the stricture even after the six weeks' rest, the patient being obliged all the time to wear a tube up his rectum; thirdly, it is stated that this operation is often the cause of a serious, because in tractable, ulceration of the rectum;* and, lastly, this operation may leave a fistula not curable except by operative procedures. Syme's operation for impermeable urethra is open to the gravest of all objections-it involves a laceration of the urethra, and I should decline to perform it. If the teachings of surgery and pathology are worth anything, they tell us that the very worst form of contraction follows a laceration of the urethra; and it matters not whether the laceration be the result of accident or caused by the surgeon, the result is similar, it differs only in degree. Again, Syme's operation necessitates the use of a fistula, and if there be none such the operation is not applicable.

Now for a few words regarding that grand old French operation, "la boutonnière," compared with which lithotomy is but child's play. It is but little known in England, and, so far as $I$ know, it is in Leeds only that it is appreciated. The great advantage it possesses is that it attacks the disease at its seat and cures it, so far as any stricture can be cured. It is a rational operation, and is specially indicated. It is not a dangerous operation, and is not so diffcult of execution as is supposed, for the portion of the urethra behind the stricture is always greatly distended, so that a dissection through the perineum for a depth of one inch and a half will nearly always be sufficient. Formerly I used metal instruments with which to perform this operation; now, however, I have simplified matters, and employ only a knife and a large olivary elastic catheter. My first object is to get into the bladder from the perineum. Having made my incision down the penile urethra, I pass the catheter into the wound, and with great gentleness endeavour to insinuate it into the membranous urethra. I have never failed to effect my object. Afterwards I pass the same catheter as far as it will go, and then by cutting on its point I liberate it and pass it into the wound, from whence I conduct it along the tip of my left forefinger into the bladder. I now never leave a catheter in the bladder, but content myself with passing it every other day at first, and twice a week after the first month. At the expiration of two months it will suffice to introduce the catheter once a week, and at a later period still less frequently. For some time after the operation all the urine comes by the wound, but it usually heals up without any trouble. The great advantages which this operation possesses over all others is that it attacks the disease at its seat and does not injure any healthy part; it gives a free vent for all abscesses, and, by restoring the urethral canal, it finally cures both stricture and fistulie. In the whole range of surgery there exists not an operation which can render such signal services to the sufferer.

* From inquiry I find this objection is doubtful.

New Sydenham Society. - The annual meeting of the New Sydenham Society will be held in King's College on Friday, August 8th, at 9 A.M., when the report and balance-sheet for 1872 will be read, the office-bearers for 1873-74 elected, and other business transacted. 